\title{
TU/e EmonOWEN

\section{Multi relaxation time lattice Boltzmann simulations of multiple component fluid flows in porous media}

\section{Citation for published version (APA):}

Schmieschek, S., Narváez Salazar, A., \& Harting, J. (2013). Multi relaxation time lattice Boltzmann simulations of multiple component fluid flows in porous media. In W. E. Nagel, D. H. Kröner, \& M. M. Resch (Eds.), High Performance Computing in Science and Engineering '12: Transactions of the High Performance Computing Center, Stuttgart (HLRS) 2012 (pp. 39-49). Springer. https://doi.org/10.1007/978-3-642-33374-3_5

DOI:

10.1007/978-3-642-33374-3_5

Document status and date:

Published: 01/12/2013

\section{Document Version:}

Publisher's PDF, also known as Version of Record (includes final page, issue and volume numbers)

\section{Please check the document version of this publication:}

- A submitted manuscript is the version of the article upon submission and before peer-review. There can be important differences between the submitted version and the official published version of record. People interested in the research are advised to contact the author for the final version of the publication, or visit the $\mathrm{DOI}$ to the publisher's website.

- The final author version and the galley proof are versions of the publication after peer review.

- The final published version features the final layout of the paper including the volume, issue and page numbers.

Link to publication

\section{General rights}

Copyright and moral rights for the publications made accessible in the public portal are retained by the authors and/or other copyright owners and it is a condition of accessing publications that users recognise and abide by the legal requirements associated with these rights.

- Users may download and print one copy of any publication from the public portal for the purpose of private study or research.

- You may not further distribute the material or use it for any profit-making activity or commercial gain

- You may freely distribute the URL identifying the publication in the public portal.

If the publication is distributed under the terms of Article 25fa of the Dutch Copyright Act, indicated by the "Taverne" license above, please follow below link for the End User Agreement:

www.tue.nl/taverne

Take down policy

If you believe that this document breaches copyright please contact us at:

openaccess@tue.nl

providing details and we will investigate your claim. 


\title{
Multi Relaxation Time Lattice Boltzmann Simulations of Multiple Component Fluid Flows in Porous Media
}

\author{
Sebastian Schmieschek, Ariel Narváez, and Jens Harting
}

\begin{abstract}
The flow of fluid mixtures in complex geometries is of practical interest in many fields, ranging from oil recovery to freeze-dried food products. Due to its inherent locality the lattice Boltzmann method allows for straightforward implementation of complex boundaries and excellently scaling parallel computations. The widely applied Bhatnagar Gross Krook (BGK) scheme, used to model the contribution of particle collisions to the velocity field, does however suffer from limitiations in precision that become more prominent with increasing surface to volume ratio. To increase the accuracy of simulations of mixtures in porous media, we integrated a so-called multi relaxation time (MRT) collision scheme with a pseudo-potential method for fluids with multiple components. We describe some optimisation details of the implementation and present test results verifying the physical accuracy as well as benchmarks obtained on the XC2 Opteron cluster at the Scientific Supercomputing Centre Karlsruhe (SSCK).
\end{abstract}

\section{Introduction}

Fluid flows involving multiple components and phases are of interest in many different fields of application, e.g. oil recovery [1], soil treatment and decontamination [2], food processing [3] and fuel cell optimisation [4]. This has motivated ongoing theoretical [5] and experimental [6] as well as numerical [7-12] research.

\footnotetext{
S. Schmieschek · A. Narváez $\cdot$ J. Harting $(\bowtie)$

Institute for Computational Physics, University of Stuttgart, Pfaffenwaldring 27,

70569 Stuttgart, Germany

Department of Applied Physics, Eindhoven University of Technology, Den Dolech 2, 5600MB Eindhoven, The Netherlands

e-mail: jens@icp.uni-stuttgart.de
} 
An important measure to describe the behaviour of fluid flows in porous media is the permeability. It is relating an externally applied mean pressure gradient to a resulting mean flow rate through the medium.

Numerous numerical models have been utilized to model flow in porous media, exhibiting strengths in different areas [7-9, 13]. While finite element methods (FEM) allow for a very efficient calculation of certain systems, the incorporation of complex boundary conditions may become very tedious. Particulate models such as dissipative particle dynamics (DPD) or molecular dynamics (MD) ease the integration of various fluid components but require time averaging to produce strong statistics. Furthermore, microscopic modeling as in MD does not allow to reach length- and time-scales relevant for the simulation of porous media. While in direct comparison of algorithmic costs to macroscopic (FEM) and other mesoscopic methods (DPD), lattice Boltzmann methods (LBM) seem to be expensive, their inherent thermostatistics supresses noise in the results and thus makes the method very competititve. In adition, the localised nature of the model eases parallelization as well as the integration of complex boundary geometries.

In the decades since its introduction, the LBM has been subject to several extensions, e.g. to account for multiphase flows [16-18], thermal transport phenomena $[19,20]$ or reactive flows [21]. Furthermore weaknesses of the model were discovered and accounted for [22,23]. One prominent issue of the widely used Bhatnagar Gross Krook (BGK) collision scheme is the unphysical dependence of the position of simple bounce back boundaries on the relaxation time parameter $\tau$. While in straight channel geometries, the thusly introduced error is easy to account for, in complex boundary geometries a significant relaxation time dependent error is observed [12,23]. Amongst several other improvements, the introduction of a socalled mutlti relaxation time (MRT) collision scheme to the LB algorithm allows to mend this shortcoming.

Since many flow situations in porous media involve multiple fluid phases or components, a consistent algorithm which takes phase separation or interfacial tension into account is required. In this contribution we report aspects of the integration of a MRT collision scheme with our pseudo-potential multiphase lattice Boltzmann implementation $l b 3 d$ and present results of ongoing physics- and performance benchmarks towards a more acurrate and precise simulation tool for multiphase flows in porous media. Figure 1 shows example visualisations of our test cases: on the left hand side a rendering of the model porous medium, a BCC sphere packing and on the right hand side three snapshots of the domains of two immiscible fluids in a ternary mixture for different concentrations of an amphiphilic species.

\section{Simulation Method}

The lattice Boltzmann equation

$$
\underbrace{f_{k}\left(\mathbf{x}+\mathbf{c}_{k} \Delta t, t+\Delta t\right)-f_{k}(\mathbf{x}, t)}_{\text {Advection }}=\underbrace{\Omega\left(f_{k}, f_{k}^{\text {eq }}\right)}_{\text {Collision }}
$$



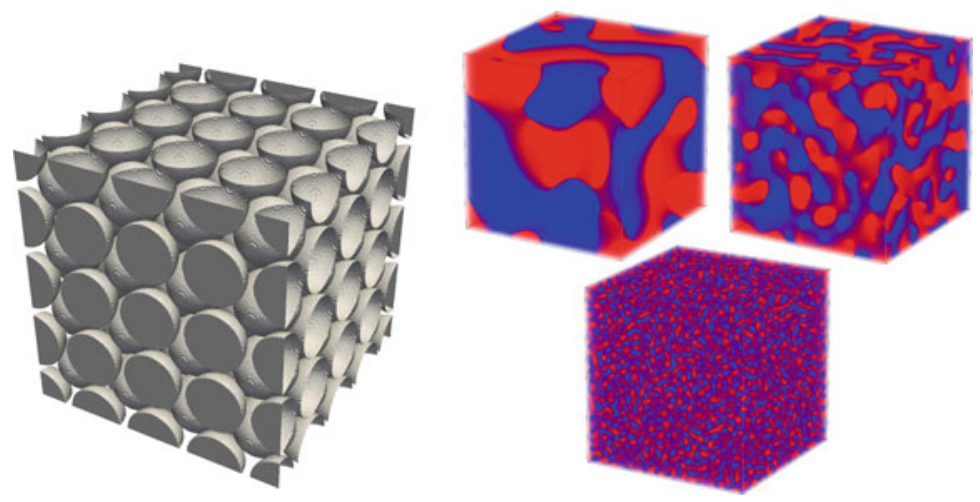

Fig. 1 Left: Rendering of the surface area of the model porous medium used in our simulations. A body centred cubic (BCC) sphere packing. The permeability $\kappa$ of this system is analytically accessible $[14,15]$. Right: Rendering of the dynamics of the spinodal decomposition of two immiscible components in a ternary mixture. Depicted is the state reached after simulation of 10,000 timesteps with surfactant concentrations of $C_{s}=\{0,18.75,31.25 \%\}$

is modeling fluid flows in terms of the single particle distribution function $f$. It is discretised on a lattice with velocities $\mathbf{c}_{k}, k=0 . . b$. Our model relies on the so-called D3Q19 lattice, comprised by $b=18$ velocities in direction of the face and edgecenters of a three dimensional cube, as well as an additional zero-velocity. The time development of $f$ is described by an undisturbed linear motion (advection) and an operator $\Omega$ representing particle interactions. The time step $\Delta t$ and the length of the lattice constant $\Delta x$ are further on chosen to equal one. The expression $f_{k}^{\text {eq }}$ designates the local equilibrium distribution, depending on the conserved properties density $\rho$ and mean velocity $\mathbf{u}$. Its form can be chosen depending on the problem to simulate. In the scope of this work a third order expansion of the local Maxwell distribution with the lattice speed of sound $c_{s}=1 / \sqrt{3}$ and lattice weights $w_{k}$ is used [24]:

$$
f_{k}^{\mathrm{eq}}=w_{k} \rho\left[1+\frac{(\mathbf{c u})}{c_{s}^{2}}+\frac{(\mathbf{c u})^{2}}{2 c_{s}^{4}}-\frac{\mathbf{u u}}{2 c_{s}^{2}}+\frac{(\mathbf{c u})^{3}}{6 c_{s}^{6}}-\frac{\mathbf{u u}(\mathbf{c u})}{2 c_{s}^{4}}\right]
$$

For the BGK approach the collision operator

$$
\Omega_{\mathrm{BGK}}=-\frac{1}{\tau}\left[f_{k}(\mathbf{x}, t)-f_{k}^{\mathrm{eq}}(\mathbf{x}, t)\right],
$$

with a single relaxation time $\tau$ is used. The relaxation time is determining the kinematic viscosity $v=c_{S}^{2}\left(\tau-\frac{1}{2}\right)$. With the above described choices the equation of state (EOS) $P=\sum_{k} f_{k}^{\text {eq }} c_{S}^{2}=\rho c_{S}^{2}=\rho k_{B} T$ of an ideal gas with density $\rho$ and Temperature $T$ is recovered, where $k_{B}$ denotes the Boltzmann constant. 
While this formulation readily allows to solve the Navier-Stokes equations up to second order accuracy, there exist several limitations to the model. Due to the single relaxation time scale the Prandtl number, i.e. the ratio of momentum to energy transport, is fixed. In the low viscosity regime $\tau \rightarrow 0.5$ strong numerical instabilities are observed. Furthermore, the position of a bounce-back (no-slip) boundary is viscosity dependent.

These issues can be adressed by introducing a different collision operator, allowing to vary the Prandtl number and increasing the overall numerical stability. The collision operator of the generalised (MRT) LBM can be written as [25]

$$
\Omega_{\mathrm{MRT}}=-\mathcal{M}^{-1} \hat{\mathcal{S}} \mathcal{M}\left[|f(\mathbf{x}, t)\rangle-\left|f^{\mathrm{eq}}(\mathbf{x}, t)\right\rangle\right] .
$$

Herein $\mathcal{M}$ is an invertible transformation matrix, relating the stochastical moments of the single particle velocity distribution $f$ to linear combinations of its discrete components $f_{k}$. It can be obtained by a Gram Schmidt orthogonalization of a matrix representation of the stochastical moments. The latter can be related to physical properties such as density $\rho$, velocity $\mathbf{u}$, momentum $\mathbf{p}$, energy $e$, etc. The collision process is performed in the space of moments, where $\hat{\mathcal{S}}$ is a diagonal matrix of the individual relaxation times. Additional to the shear viscosity, the bulk viscosity $\xi=\frac{2}{3} c_{s}^{2}\left(\frac{1}{\tau_{\text {bulk }}}-\frac{1}{2}\right)$ can be varied.

The Shan Chen pseudo-potential method alters the local mean velocity

$$
\mathbf{u}=\frac{\sum_{\alpha}\left(\mathbf{u}_{\alpha} \cdot \rho_{\alpha}\right) / \tau_{\alpha}}{\sum_{\alpha}\left(\rho_{\alpha}\right) / \tau_{\alpha}}+\frac{F^{\alpha} \cdot \tau_{\alpha}}{m_{\alpha}},
$$

common to all species $\alpha$ of density $\rho$ and mass $m$ by introducing a phenomenological force

$$
\mathbf{F}^{\alpha \bar{\alpha}}=\frac{d p^{\alpha}}{d t}(\mathbf{x})=-\psi^{\alpha}(\mathbf{x}) \sum^{\alpha} g_{\alpha \bar{\alpha}} \sum_{k=0}^{b} \psi^{\bar{\alpha}}\left(\mathbf{x}^{\prime}\right) \mathbf{c}_{k},
$$

which is defined to be proportional to a functional of the fluid densities $\psi^{\alpha}=1-e^{-\rho}$ and a coupling parameter $g_{\alpha \bar{\alpha}}$ determining the nature and magnitude of interaction between the components $\alpha$ and $\bar{\alpha}$. This accounts for additional (non-ideal) terms in the equation of state $[16,26]$. For attractive self-interactions $\left(g_{\alpha \alpha}<0\right)$ of a magnitude larger than a critical value $g_{\mathrm{c}}$ a phase separation in a vapour and a liquid phase can be observed. Repulsive interactions between two components $\left(g_{\alpha \bar{\alpha}}>0\right)$ are utilised to model systems of partly miscible or immiscible fluid mixtures. While the input parameters are determined strictly phenomenological, this approach has recently been shown to be equivalent to the explicit adjustment of the free energy of the system [27].

Amphiphiles (surfactants) are comprised of a hydrophilic (water-loving) head group and a hydrophobic (oil-loving) tail. Amphiphilic behaviour is modeled by a dipolar moment $\mathbf{d}$ with orientation $\vartheta$ defined for each lattice site. It is relaxing in a BGK-like process, where the equilibrium moment is dependent on the surrounding 
fluid densities [28]. The introduction of the dipole vector accounts for three additional Shan Chen type interactions, namely an additional force-term

$$
\mathbf{F}^{\alpha s}=-2 \psi^{\alpha}(\mathbf{x}, t) g_{\alpha s} \sum_{k \neq 0} \tilde{\mathbf{d}}\left(\mathbf{x}+\mathbf{c}_{k}, t\right) \cdot \boldsymbol{\Theta}_{k} \psi^{s}\left(\mathbf{x}+\mathbf{c}_{k}, t\right)
$$

for the regular fluid components $\alpha$ imposed by the surfactant species $s$. Therein, the tilde denotes post collision values and the second rank tensor $\boldsymbol{\Theta}_{k} \equiv \mathbb{I}-3 \frac{\mathbf{c}_{k} \mathbf{c}_{k}}{c^{2}}$, with the identity operator $\mathbb{I}$ weights the dipoles force contribution according to the orientation relative to the density gradient. The surfactant species is subject to forcing as well, where the contribution of the regular components $\alpha$ is given by

$$
\mathbf{F}^{\alpha s}=2 \psi^{s}(\mathbf{x}, t) \tilde{\mathbf{d}}(\mathbf{x}, t) \cdot \sum_{\alpha} g_{\alpha s} \sum_{k \neq 0} \boldsymbol{\Theta}_{k} \psi^{\alpha}\left(\mathbf{x}+\mathbf{c}_{k}, t\right),
$$

and

$$
\begin{gathered}
\mathbf{F}^{s s}=-\frac{12}{\left\|\mathbf{c}_{s}\right\|^{2}} \psi^{s}(\mathbf{x}, t) g_{s s} \cdot \sum_{k \neq 0} \psi^{s}\left(\mathbf{x}+\mathbf{c}_{k}, t\right) \cdot\left(\tilde{\mathbf{d}}\left(\mathbf{x}+\mathbf{c}_{k}, t\right) \cdot \boldsymbol{\Theta}_{k} \cdot \tilde{\mathbf{d}}(\mathbf{x}, t) \mathbf{c}_{k}\right. \\
\left.+\left[\tilde{\mathbf{d}}\left(\mathbf{x}+\mathbf{c}_{k}\right) \tilde{\mathbf{d}}(\mathbf{x}, t)+\tilde{\mathbf{d}}(\mathbf{x}, t) \tilde{\mathbf{d}}\left(\mathbf{x}+\mathbf{c}_{k}, t\right)\right] \cdot \mathbf{c}_{k}\right)
\end{gathered}
$$

is the force due to self-interaction of the amphiphilic species [29].

\section{Implementation}

The lattice Boltzmann implementation $l b 3 d$ provides functionality to simulate threedimensional simple, binary immiscible and ternary amphiphilic fluid systems using the Shan Chen pseudo-potential model for non-ideal fluid interactions.

The boundary conditions available include periodic boundaries, body forcing, and bounce-back boundaries as well as Lees-Edwards shearing for simple and binary fluid mixtures. The software is written in Fortran 90 and parallelized using MPI. It supports XDR and parallel HDF5 formats for I/O and provides checkpoint and restart for long-running simulations.

The code has been developed at University College London, University of Stuttgart and Eindhoven University of Technology. It has been ported to many supercomputers worldwide, where it has shown excellent scalability. Recently it has been shown to scale linearly on up to 262,144 cores on the European Blue Gene/P system Jugene [30]. We were able to confirm very good scaling of our ternary systems on the Opteron based XC2 as well (Fig. 2, left). 


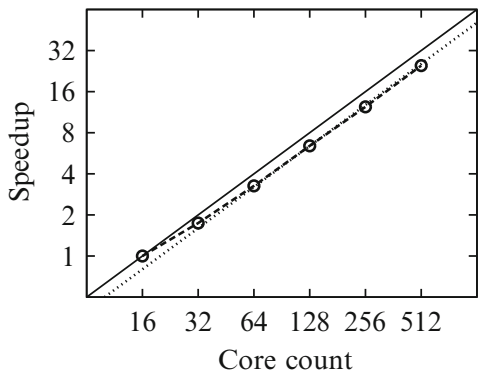

\begin{tabular}{|c|c|c||r|r|r|}
\hline \multicolumn{2}{|c||}{ Components } & $\Omega$ & kLUPS & Difference \\
\hline $\mathrm{a}$ & $\mathrm{b}$ & $\mathrm{s}$ & & & \\
\hline $\mathrm{X}$ & & & BGK & 1450 & \\
\hline $\mathrm{x}$ & & & MRT & 1214 & $16.3 \%$ \\
\hline $\mathrm{x}$ & $\mathrm{x}$ & & BGK & 383 & \\
\hline $\mathrm{x}$ & $\mathrm{x}$ & & MRT & 357 & $6.9 \%$ \\
\hline $\mathrm{x}$ & $\mathrm{x}$ & $\mathrm{x}$ & $\mathrm{BGK}$ & 155 & \\
\hline $\mathrm{x}$ & $\mathrm{x}$ & $\mathrm{x}$ & $\mathrm{MRT}$ & 146 & $5.8 \%$ \\
\hline
\end{tabular}

Fig. 2 Left: Speedup measure for a ternary system of $256^{3}$ lattice sites on the XC2 at SSC Karlsruhe. The solid line represents ideal scaling relative to 16 core performance, the dotted line represents ideal scaling relative to 128 core performance. We observe very good scaling. Right: Performance comparison, measured in thousand lattice updates per second (kLUPS) produced on a single processor core based on the Intel Nehalem microarchitechture, running at a clock rate of $2.4 \mathrm{GHz}$. Results for a variation in component count using a BGK or MRT collision scheme, respectively. The components $a$ and $b$ denote immiscible Newtonian fluids, whereas component $s$ is an amphiphilic fluid species. With increasingly complex fluid interaction calculations, the contribution of a more costly collision scheme is rendered less important

In recent years, $l b 3 d$ has been coupled to a molecular dynamics solver in order to simulate complex fluids containing particulate components, such as blood [31], or nano-particle stabilised emulsions and suspensions [32]. Moreover, the code has been used to study for example the self-assembly of cubic mesophases [33], micromixing [34], flow through porous media [10] and fluid surface interactions $[35,36]$. A refactored version of the code with limited functionality that focuses mainly on multiphase fluid simulation functionalities, has recently been released under the LGPL [37].

In order to improve the accuracy and numerical stability of $l b 3 d$ with respect to the application to the simulation of multiphase flows in porous media, recently a MRT collision model was integrated with the software. While the MRT collision algorithm is more complex than the BGK collision scheme and can cause significant performance loss when implemented naively, the increase in calculation cost can be dramatically reduced. We take advantage of two properties of the system to minimize the impact of the additional MRT operations on the code performance:

1. The symmetry of the lattice allows to precalculate the sum and difference of discrete velocities which are linear dependent, thus saving at least half of the calculation steps [38].

2. The equilibrium stochastical moments can be expressed as functions of the conserved properties density and velocity, thus saving the transformation of the equilibrium distributions [25].

As such, the performance penalty could be reduced below $17 \%$, which is close to the minimal additional cost reported in [25]. Since in multiphase systems the relative cost of the collision scheme is further reduced, the use of the MRT scheme has even less impact on the performance (Fig. 2, right). 

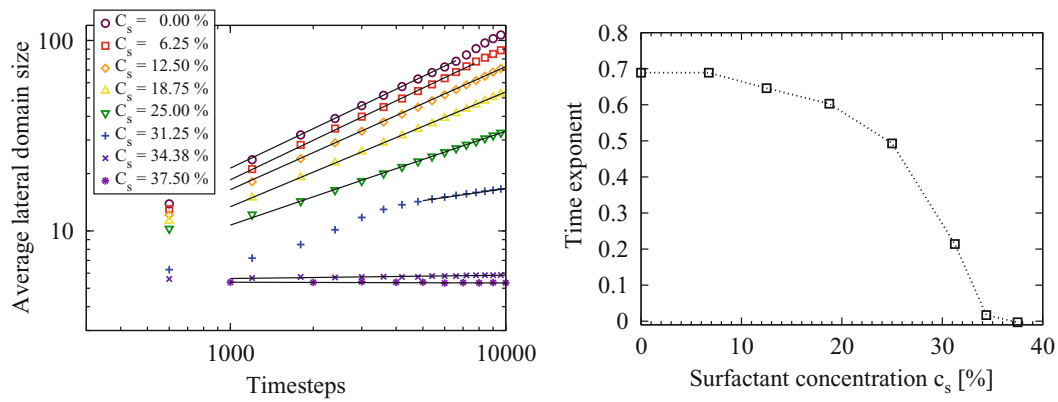

Fig. 3 Left: Double logarithmic plot of the dynamics of the average domain size for different surfactant concentrations. After an initial phase, resulting from the chosen homogenous random initialisation of the fluid densities, the average domain size follows a power-law. Increasing surfactant concentration is slowing down the domain growth. With exceeding a concentration of $\mathrm{C}_{s} \approx 35 \%$ the critical micelle concentration is reached and a stable microemulsion with a minimal domain size is formed. Right: Time exponent of the dynamics of the average domain size as a function of the surfactant concentration, determined by a fitted power-law behaviour depicted on the left-hand side. At low concentrations $\mathrm{C}_{s} \lesssim 10 \%$ the impact of added surfactant on the domain size dynamics is close to negligible. As the concentration approaches the saturation of the interface the retardation of the decomposition becomes very sensitive to added surfactant

\section{Results}

To evaluate the functionality of the newly implemented MRT collision scheme, simulations of the influence of surfactant concentration on the decomposition process of two immiscible fluid components, earlier performed with $l b 3 d$ utilising the BGK method are reproduced [33].

While keeping the total density of all fluid components constant at $\rho_{\text {tot }}=0.8-$ equivalent to constant pressure - the concentration of the surfactant component is varied between 0 and $37.5 \%$ in steps of $6.25 \%$. Simulations are carried out on a domain of $256^{3}$ lattice sites. The coupling parameter between the immiscible components $b$ (blue) and $r$ (red) is set to $g_{b r}=0.08$. The coupling parameters to the surfactant species are set to $g_{\alpha s}=-0.006$ and $g_{s s}=-0.003$, respectively. The fluid components are initialised at homogenous density distribution and a random dipole orientation. The average domain size is extracted by evaluation of the Fourier transformation of the field of the order parameter $\Phi=\rho_{b}-\rho_{r}$ which becomes zero at the interface of the immiscible components [33].

As can be observed from Fig. 3, two regimes of the dynamics can be distinguished. At low to intermediate surfactant concentrations $\left(\mathrm{C}_{s}<35 \%\right)$ spinodal decomposition is taking place, resulting in completely demixed fluids. Here, after an initial phase related to the formation of domains from the homogenous initialised fluids, the average domain size clearly follows a power-law with respect to time. Figure 3 (left) shows a double-logarithmic plot of the dynamics of the average domain sizes for varying surfactant concentration. The extracted numerical values for the power-law relation can be found in Fig. 3 (right). Average domain sizes 
exceeding 80 lattice sites have been excluded from the fit, as in this range the periodic boundary conditions begin to influence the results. In the range of higher concentration close to the critical micelle concentration $\left(\mathrm{C}_{s}=31.25 \%\right)$ the initial phase is extended considerably as compared to lower concentrations. The impact of adding surfactant on the dynamics of the average domain size is almost an order of magnitude large here as compared to the low concentration range around $10 \%$. With exceeding a concentration of $\mathrm{C}_{s} \approx 35 \%$ the critical micelle concentration is reached and stable microemulsions with a constant domain size are formed. These results are in agreement with the findings of the reference study and allow confidence in the MRT collision scheme.

To test the behaviour of the MRT scheme in a complex geometry, the flow of two miscible phases through the model BCC sphere packing porous medium, illustrated in Fig. 1 (left), is simulated. The permeability for this model system

$$
\kappa=\frac{a^{2}}{6 \pi \chi h}
$$

can be derived analytically in terms of the side length of the unit cell $a$, the dimensionless drag $h$ and a geometry-factor $\chi$ giving the ratio of actual to maximal sphere radius. We choose the maximum radius here, so $\chi=1$. The dimensionless drag can be shown to depend on the system geometry only and can be expressed as an expansion of $\chi[14,15]$.

In our simulation setup, we measure the permeability using Darcy's law

$$
\kappa=-\rho v \frac{\langle u\rangle}{\langle\nabla P\rangle},
$$

with the fluid density $\rho$, the kinematic viscosity $v$, the average velocity $\langle u\rangle$ and the average pressure gradient $\langle\nabla P\rangle$. For the test runs two miscible fluids of equal density $\rho_{r}=\rho_{b}=1.0$ and equal kinematic viscosity, varied by the relaxation parameter $\tau$, are driven through the porous medium by applying a body force of $F=0.001$ in lattice units in a four lattice sites long layer located in the center of a buffer region of 20 lattice sites width.

Figure 4 shows a comparison of absolute permeability measurements conducted in simulations with varying relaxation parameter $\tau$ using the MRT and BGK collision scheme, respectively. In these preliminary simulations the only relaxation parameter modified in the MRT scheme is the one related to the dynamic viscosity, all other parameters are kept to equal 1 or 0 for parameters related to conserved properties ( $\rho$ and $\mathbf{u}$ ). In this configuration at $\tau=1$, the MRT scheme is therefore equivalent to the BGK scheme, as can be observed from the identical outcome of the permeability measure at that point. However, already for this parameter set, the dependence of the system's behaviour on the relaxation parameter ist less pronounced. It has been shown that this dependence can be eliminated by a suitable choice of parameters [23]. However, previous attempts by other groups were not combined with the Shan Chen multicomponent method and thus did not allow to 


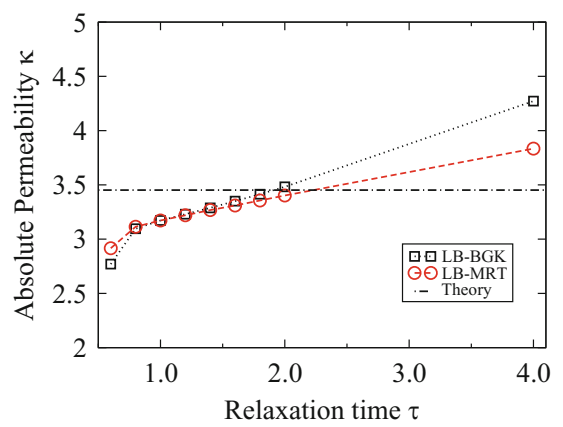

Fig. 4 Preliminary results of measurements of absolute permeability of the model BCC sphere packing porous medium for varying relaxation times $\tau$. In this case we use non-optimised values for the MRT relaxation parameters, all equal to 1 . In the case of $\tau=1.0$, this reduces the MRT to the BGK-scheme as is here illustrated by identical results. By adjusting the relaxation parameter set, the dependence of the permeability measurement on the relaxation parameter can be completely resolved [23]. Since equal fluid properties were chosen, the relative permeability $\kappa_{\alpha} / \kappa$ is 0.5 for all cases

simulate multicomponent flow in porous media. All relative permeabilities $\kappa_{\alpha} / \kappa$ measured were found to be exactly 0.5 which is to be expected, since in the runs presented here all properties of the liquid components were set to be equal.

These promising results allow to proceed with further tests, such as to optimise the MRT parameter set and study the accuracy of relative permeability measurements for fluid mixtures with different properties. Possible future applications of this model include then permeability calculations for ternary amphiphilic mixtures and studies of imbibition and demixing processes in porous media, where our model can take effects of wettability into account as well.

\section{Summary}

To improve accuracy and efficiency of the application of our lattice Boltzmann implementation for fluid flows with multiple components in porous media we have implemented a multi relaxation time collision scheme. The steps taken to optimize the performance have allowed to limit the increase in calculation time close to the expected minimum. Furthermore we could show that for multi-component systems the additional calculation time spent in the collision step is very small in comparison to the time increase due to the calculation of the interaction forces. The reproduction of prior simulations of the dynamics of an amphiphilic fluid mixture as well as a currently modest but optimisable improvement in accuracy on a broader scale of viscosity values allow confidence in the implementation. Preliminary tests of permeability measurements for multicomponent flows in porous media are promising and allow to proceed further towards productive application of the model to the simulation of ternary amphiphilic mixtures in porous media. 
Acknowledgements This work was financed within the DFG priority program "nano- and microfluidics" and by the NWO/STW VIDI grant of J. Harting. Sebastian Schmieschek likes to express his gratitude to the HPC-Europa2 programme for funding during development. We thank the Scientific Supercomputing Center Karlsruhe for providing the computing time and technical support for the presented work.

\section{References}

1. L. Paterson. Diffusion-limited aggregation and two-fluid displacements in porous media. Phys. Rev. Lett., 52:1621-1624, Apr 1984.

2. Z. Zhang and J. E. Smith. The velocity of dnapl fingering in water-saturated porous media: laboratory experiments and a mobile-immobile-zone model. Journal of Contaminant Hydrology, 49(3-4):335-353, 2001.

3. A. Datta. Porous media approaches to studying simultaneous heat and mass transfer in food processes. i: Problem formulations. Journal of Food Engineering, 80(1):80-95, 2007.

4. Z. Wang, C. Wang, and K. Chen. Two-phase flow and transport in the air cathode of proton exchange membrane fuel cells. Journal of Power Sources, 94(1):40-50, 2001.

5. R. de Boer. Highlights in the historical development of the porous media theory: Toward a consistent macroscopic theory. Applied Mechanics Reviews, 49(4):201-262, 1996.

6. R. Lenormand, E. Touboul, and C. Zarcone. Numerical models and experiments on immiscible displacements in porous media. Journal of Fluid Mechanics, 189:165-187, 1988.

7. M. Liu, P. Meakin, and H. Huang. Dissipative particle dynamics simulation of pore-scale multiphase fluid flow. Water Resour. Res., 43(4):W04411, April 2007.

8. X. Yi, J. Ghassemzadeh, K. Shing, and M. Sahimi. Molecular dynamics simulation of gas mixtures in porous media. I. Adsorption. J. Chem. Phys., 108(5):2178-2188, Feb 1998.

9. T. Y. Hou and X.-H. Wu. A multiscale finite element method for elliptic problems in composite materials and porous media. Journal of Computational Physics, 134(1):169-189, 1997.

10. J. Harting, M. Venturoli, and P. V. Coveney. Large-scale grid-enabled lattice-Boltzmann simulations of complex fluid flow in porous media and under shear. Phil. Trans. R. Soc. Lond. A, 362:1703-1722, 2004.

11. A. Narváez and J. Harting. A D3Q19 lattice-Boltzmann pore-list code with pressure boundary conditions for permeability calculations. Advances in Applied Mathematics and Mechanics, $2: 685,2010$.

12. A. Narváez, T. Zauner, F. Raischel, R. Hilfer, and J. Harting. Quantitative analysis of numerical estimates for the permeability of porous media from lattice-Boltzmann simulations. J. Stat. Mech: Theor. Exp., 2010:P211026, 2010.

13. J. Harting, T. Zauner, A. Narvaez, and R. Hilfer. Flow in porous media and driven colloidal suspensions. In W. Nagel, D. Kröner, and M. Resch, editors, High Performance Computing in Science and Engineering '08. Springer, 2008.

14. H. Hasimoto. On the periodic fundamental solutions of the Stokes equations and their application to viscous flow past a cubic array of spheres. Journal of Fluid Mechanics, 5(02):317-328, 1959.

15. A. Sangani and A. Acrivos. Slow flow through a periodic array of spheres. International Journal of Multiphase Flow, 8(4):343-360, 1982.

16. X. Shan and H. Chen. Lattice Boltzmann model for simulating flows with multiple phases and components. Phys. Rev. E, 47(3):1815, 1993.

17. M. R. Swift, W. R. Osborn, and J. M. Yeomans. Lattice Boltzmann simulation of nonideal fluids. Phys. Rev. E, 75(5):830, 1995.

18. A. K. Gunstensen, D. H. Rothman, S. Zaleski, and G. Zanetti. Lattice Boltzmann model of immiscible fluids. Phys. Rev. A, 43(8):4320, 1991. 
19. B. Dünweg, U. D. Schiller, and A. J. C. Ladd. Statistical mechanics of the fluctuating lattice Boltzmann equation. Phys. Rev. E, 76:36704, 2007.

20. R. Adhikari, K. Stratford, M. E. Cates, and A. J. Wagner. Fluctuating lattice Boltzmann. Europhys. Lett., 71:473, 2005.

21. Q. Kang, P. C. Lichtner, and D. Zhang. Lattice Boltzmann pore-scale model for multicomponent reactive transport in porous media. J. Geophys. Res., 111(B5):B05203, May 2006.

22. X. He, Q. Zou, L.-S. Luo, and M. Dembo. Analytic solutions of simple flows and analysis of nonslip boundary conditions for the lattice Boltzmann BGK model. Journal of Statistical Physics, 87:115-136, 1997.

23. C. Pan, L.-S. Luo, and C. T. Miller. An evaluation of lattice Boltzmann schemes for porous medium flow simulation. Computers \& Fluids, 35(8-9):898-909, 2006.

24. S. Succi. The lattice Boltzmann equation for fluid dynamics and beyond. Oxford University Press, 2001.

25. D. d'Humières, I. Ginzburg, M. Krafczyk, P. Lallemand, and L. Luo. Multiple-relaxation-time lattice Boltzmann models in three dimensions. Phil. Trans. R. Soc. A, 360:437451, 2002.

26. X. Shan and G. Doolen. Multicomponent lattice-Boltzmann model with interparticle interaction. J. Stat. Phys., 81(112):379, 1995.

27. M. Sbragaglia and X. Shan. Consistent pseudopotential interactions in lattice Boltzmann models. Phys. Rev. E, 84:036703, Sep 2011.

28. M. Nekovee, P. V. Coveney, H. Chen, and B. M. Boghosian. Lattice-Boltzmann model for interacting amphiphilic fluids. Phys. Rev. E, 62(6):8282-8294, Dec 2000.

29. M. Nekovee, P. V. Coveney, H. Chen, and B. M. Boghosian. Lattice-Boltzmann model for interacting amphiphilic fluids. Phys. Rev. E, 62:8282, 2000.

30. D. Groen, O. Henrich, F. Janoschek, P. Coveney, and J. Harting. Lattice-boltzmann methods in fluid dynamics: Turbulence and complex colloidal fluids. In W. F. Bernd Mohr, editor, Jülich Blue Gene/P Extreme Scaling Workshop 2011. Jülich Supercomputing Centre, 52425 Jülich, Germany, apr 2011. FZJ-JSC-IB-2011-02; http://www2.fz-juelich.de/jsc/docs/autoren2011/ mohr1/.

31. F. Janoschek, F. Toschi, and J. Harting. Simplified particulate model for coarse-grained hemodynamics simulations. Phys. Rev. E, 82:056710, 2010.

32. F. Jansen and J. Harting. From Bijels to Pickering emulsions: a lattice Boltzmann study. Phys. Rev. E, 83:046707, 2011.

33. N. González-Segredo, J. Harting, G. Giupponi, and P. V. Coveney. Stress response and structural transitions in sheared gyroidal and lamellar amphiphilic mesophases: lattice-Boltzmann simulations. Phys. Rev. E, 73:031503, 2006.

34. A. Sarkar, A. Narváez, and J. Harting. Quantification of the degree of mixing in chaotic micromixers using finite time Lyapunov exponents. Microfluidics and Nanofluidics, in press, 2010.

35. C. Kunert and J. Harting. Roughness induced apparent boundary slip in microchannel flows. Phys. Rev. Lett., 99:176001, 2007.

36. S. Schmieschek, A. V. Belyaev, J. Harting, and O. I. Vinogradova. Tensorial slip of superhydrophobic channels. Phys. Rev. E, 85:016324, 2012.

37. 'lb3d open source version 7' available online, March 2012. http://mtp.phys.tue.nl/lb3d.

38. B. Chun and A. J. C. Ladd. Interpolated boundary condition for lattice Boltzmann simulations of flows in narrow gaps. Phys. Rev. E, 75:066705, Jun 2007. 